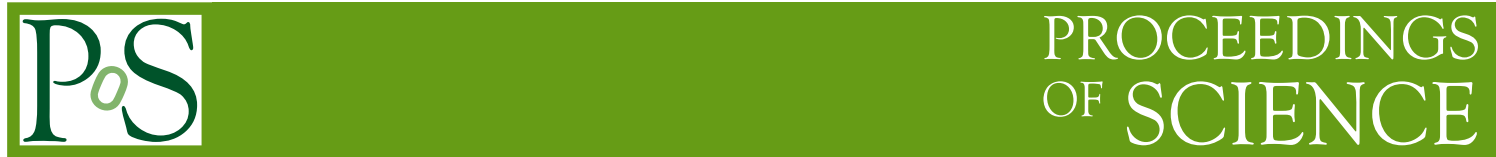

\title{
Volume independence of large-N QCD with adjoint fermions
}

\section{Barak Bringoltz and Stephen R. Sharpe*}

Physics Department University of Washington Seattle, WA 98195-1560

E-mail: barak@phys.washington.edu, sharpe@phys.washington . edu

It has been proposed that four-dimensional QCD with fermions in the adjoint representation exhibits volume-independence in the large- $N$ limit. If correct, this would mean that results for physical quantities could be obtained from the single-site version of the model. A necessary condition for volume-independence is that the $\left(Z_{N}\right)^{4}$ center-symmetry of the single-site theory is unbroken. We explore the phase diagram of the theory with a single Dirac fermion using Wilson fermions for a number of colors in the range $8 \leq N \leq 15$, and identify the region in the parameter space of quark mass and gauge coupling where the symmetry appears to be unbroken. Our evidence suggests that this region includes both light and heavy quarks, and our results are consistent with this region extending to the continuum limit.

The XXVII International Symposium on Lattice Field Theory

July 26-31, 2009

Peking University, Beijing, China

\footnotetext{
* Speaker.
} 


\section{Introduction}

Long ago, Eguchi and Kawai (EK) proposed the striking idea that the properties of gauge theories become independent of the volume when the number of colors, $N$, is sent to infinity [1]. Using a lattice regularization, this would allow one to determine physical, infinite-volume, quantities from calculations on a single lattice site, with both color and space-time degrees of freedom packed into the four link matrices. This idea is of both theoretical interest - can such matrix models indeed represent field theories? - and of possible practical utility - it might allow the determination of large- $N$ properties in a more computationally efficient way. This would in turn allow one to connect to approximate analytic approaches to large- $N$ QCD, as well as to learn about QCD itself.

The application of this "volume-reduction" to pure gauge theories turned out not to be simple. As discussed below, the $Z_{N}^{4}$ center symmetry of the $S U(N)$ volume-reduced lattice theory must be unbroken for the equivalence to infinite volume to hold, but this symmetry is, in fact, spontaneously broken by the single-site theory [2,3]. Various proposed "fixes"-in particular, "momentum-quenching" and twisting - have turned out not to work. See [4] for references to this history. What does work, however, is to reduce to a lattice whose physical extent exceeds a scale of about $1 \mathrm{fm}$ [5], and this approach, which, for a lattice spacing $a \approx 0.15 \mathrm{fm}$, allows one in practice to work on lattices of size $\sim 6^{4}$, has been successful in determining several large-volume physical quantities.

In our present work (summarized here but described in much more detail in Ref. [6]) we return to the possibility of complete volume reduction to a single-site theory, but in the context of QCDlike theories with fermions in two-index representations. In particular, our evidence suggests that such reduction holds for the theory with "quarks" in the adjoint representation. In addition, it appears that these quarks can be heavy, with $m \gg \Lambda_{\mathrm{QCD}}$, in which case the long distance theory is the pure-gauge theory. In that case the reduction we find implements the original idea of EK.

Our motivation comes from the following relations between theories. Start with QCD, with $N=3$, but consider the fundamental representation Dirac fermions $\left(2 N_{f}\right.$ of them) to be in the equivalent antisymmetric (AS) representation with two antiquark indices. Now take $N \rightarrow \infty$ and obtain the "orientifold" large- $N$ limit of QCD [7]—one in which quark-loops are not suppressed. This theory is "within $1 / N$ " of physical QCD. Next use the "orientifold" equivalence of Armoni, Shifman and Veneziano [8], which states that the even C sector of the large- $N$ theory with $2 N_{f}$ AS Dirac fermions is equivalent to the corresponding C-even sector of the large- $N$ theory with $N_{f}$ Dirac fermions in the adjoint irrep. Finally, follow Kovtun, Ünsal and Yaffe and use an orbifold equivalence to relate, when $N \rightarrow \infty$, the latter field theory to the single-site gauge theory with $N_{f}$ adjoint Dirac fermions [9]. If these equivalences hold, the large- $N$ single-site theory gives results for two interesting field theories, one of which is close to physical QCD. ${ }^{1}$

In our work to date we have taken $N_{f}=1$-so that the corresponding QCD-like theory has two degenerate quarks - but $N_{f}=1 / 2$ and 2 are also of considerable interest (the former is related to SUSY Yang-Mills and to 1-flavor QCD, the latter to the potentially nearly-conformal theory with 2 adjoint Dirac fermions).

\footnotetext{
${ }^{1}$ For details on how one extracts long-distance quantities such as the string tension and meson masses from a singlesite model see Refs. [1, 5, 6, 9].
} 
A crucial question is, of course, what conditions must be satisfied for the equivalences to hold. The necessary and sufficient conditions are as follows [10]:

1. Large- $\mathrm{N}$ factorization holds.

2. The orientifold equivalence requires that $\mathrm{C}$ is not spontaneously broken in either the theory with AS or that with Adjoint fermions, at large volumes.

3. The orbifold equivalence requires that translation invariance is unbroken in the large-volume theory with Adjoint fermions.

4. The orbifold equivalence also requires that the $Z_{N}^{4}$ center symmetry of the single-site theory with Adjoint fermions be unbroken.

The first three conditions are expected to hold (although $\mathrm{C}$ can be broken for small volumes, and translation invariance can break at nonzero density) and it is the last condition that is the weakest link. Indeed, as noted above, this is the condition that fails in the pure gauge case. Thus our initial focus has been on determining whether the last condition holds.

There are two previous calculations which suggest that the center symmetry of the single-site theory may be unbroken. Ref. [9] calculated the one-loop effective potential for Polyakov loops in continuum regularization on $R^{3} \times S^{1}$, i.e. with one direction compactified. A weak-coupling calculation is then justified if the compact direction is small enough. It was found that, if one uses periodic boundary conditions, and for massless fermions, the $Z_{N}$ center symmetry is unbroken for $N_{f}=1 / 2,1$ and 2, but broken for $N_{f}=0$. (For a recent extension to nonzero masses see Ref. [11].) In words, the fermionic contributions to $V_{\text {eff }}$ lead to a repulsion between the eigenvalues of the Polyakov loop that overcome the attraction caused by the gluonic contributions. The same has been found to be true in a lattice regularized calculation [12], although there remains some uncertainty over what happens if only the compact direction is discretized and when nontrivial forms of center symmetry breakings are studied [13]. These results are encouraging, but do not directly apply to the single-site model. Perturbation theory for this theory has severe IR divergences, and the appropriate analysis has not been done. For this reason, and because we are interested in practice in intermediate values of the coupling where one-loop perturbation theory is not accurate $(b \sim 0.33$, corresponding to $\beta \sim 6$ for $N=3$ ), we have chosen to do a non-perturbative calculation.

\section{The (possibly) equivalent theories}

We choose to use Wilson fermions and the Wilson gauge action. The (large-volume) lattice field theory then has the action

$$
S_{F T}=2 N b \sum_{\text {plaq }} \operatorname{Re} \operatorname{Tr} U_{\text {plaq }}+\bar{\psi} D_{\mathrm{W}} \psi
$$

where $U_{\text {plaq }}$ is composed, as usual, of the fundamental irrep gauge matrices, $b=\left(g^{2} N\right)^{-1}$ is the inverse 't Hooft coupling, and the fermion operator is ( $x$ and $y$ labeling lattice sites)

$$
\left(D_{W}\right)_{x y}=\delta_{x y}-\kappa\left[\sum_{\mu=1}^{4}\left(1-\gamma_{\mu}\right) U_{x, \mu}^{\mathrm{adj}} \delta_{y, x+\mu}+\left(1+\gamma_{\mu}\right) U_{x, \mu}^{\mathrm{adj} \dagger} \delta_{y, x-\mu}\right],
$$


with the links here being in the adjoint representation. There is a single adjoint Dirac fermion field, corresponding to $N_{f}=1$. Boundary conditions are periodic in all four directions on all fields.

Notable symmetries of this theory are the usual $Z_{N}^{4}$ center symmetry and an $S O(2)$ flavor symmetry. The latter can be seen by writing the action in terms of two Majorana fields.

The corresponding single-site theory is obtained simply by removing the sum over sites. One then has a theory of four link matrices $U_{\mu}$ and a single $\psi$ and $\bar{\psi}$. The fermion operator becomes

$$
D_{W}^{\mathrm{red}}=1-\kappa \sum_{\mu=1}^{4}\left[\left(1-\gamma_{\mu}\right) U_{\mu}^{\mathrm{adj}}+\left(1+\gamma_{\mu}\right) U_{\mu}^{\mathrm{adj} \dagger}\right] \text {. }
$$

This looks peculiar because the first derivative part connects back (necessarily) to the same site, and so vanishes if $U_{\mu}=1$. This part becomes non-trivial, however, since in the reduced model the $U_{\mu}$ fluctuate around nontrivial background gauge fields, and one can see in perturbation theory how space-time (or, more precisely, momentum space) becomes embedded in the link matrices.

The symmetries of the single-site theory are an odd-looking gauge symmetry $\left[U_{\mu} \rightarrow \Omega U_{\mu} \Omega^{\dagger}\right.$ for all $\mu$ with $\Omega \in S U(N)$ ] and the main player in the following - the $Z_{N}^{4}$ center symmetry:

$$
U_{\mu} \rightarrow U_{\mu} z^{n_{\mu}} \quad \text { with } \quad z=e^{2 \pi i / N} \quad \text { and } \quad n_{\mu} \in Z_{N} .
$$

It is important that the putative equivalence applies when both theories having the same bare couplings $b$ and $\kappa$. The continuum limit is to be taken after $N \rightarrow \infty$ so that (if the equivalence holds) the single-site theory has given one information about the infinite volume field theory.

In order to test the equivalence, it is useful to have some knowledge of the phase diagram in the $\kappa-b$ plane of the large-volume large- $N$ adjoint fermion theory. Since, to our knowledge, this theory has not been simulated previously for any $N$, we begin by giving an educated conjecture for the phase diagram. This is shown in the left panel of Fig. 1. The continuum limit is at the top while the pure gauge theory lies along the left axis.

The most important feature is the $\kappa_{c}$ line, which represents the line (or region of Aoki phase) along which (within which) the quarks attain their minimum mass. The presence of this line/region is predicted by chiral perturbation theory including lattice artefacts, for which the analysis is very similar to that for QCD, except that the chiral symmetry breaking pattern is here $S U(2) \rightarrow S O(2)$. For more details see Ref. [6]. Just as in QCD, one finds that, near the continuum limit, one can either have a first-order or Aoki-phase scenario. Our data (discussed below) indicate that it is the former which occurs. For very small $b$, however, the strong-coupling expansion predicts an Aoki-phase. Thus we have drawn the line becoming a region at small $b$. For large $b$ the line must approach $\kappa_{c}=1 / 8$.

The other feature is the (possible) line of bulk transitions running roughly horizontally. We know from simulations that this transition occurs in the large- $N$ pure gauge theory, and that it is strongly first order. It is not a deconfinement transition, but rather a lattice artefact. This transition must extend for some distance into the plane, but we do not know how far. Our numerical evidence turns out to be inconclusive as to whether the transition turns into a crossover as one moves into the plane. In either case, we want to work on the continuum (large $b$ ) side of the transition region to avoid lattice artefacts.

The right panel of Fig. 1 shows our conjecture for the phase diagram of the single-site model. In this case we must take $N \rightarrow \infty$ for there to be true transitions, although it turns out that this is 

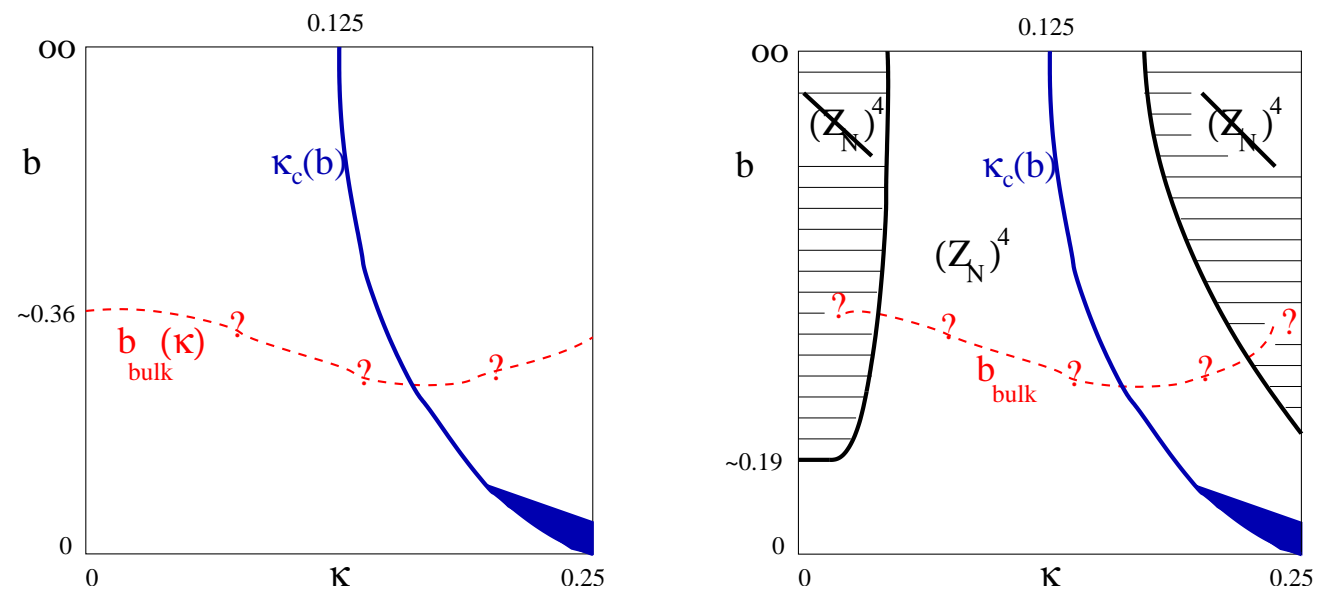

Figure 1: Conjectured large- $N$ phase diagrams for theories with a single adjoint Dirac fermion. Left panel: infinite volume; right panel: single-site.

not much of an issue in practice. The left axis (at $\kappa=0$ ) corresponds to the EK model, which has the above-noted center symmetry breaking transition (really, a sequence of transitions) at $b \sim 0.19$. Based on the perturbative calculations of Ref. [12] we expect the symmetry to be restored when the quark becomes light, and that it remains unbroken for a range of $\kappa$ including $\kappa_{c}$. Our conjecture therefore contains a funnel-shaped region of unbroken center symmetry in which volumeindependence should hold. We recall that, in the continuum limit, theories equidistant from $\kappa_{c}$ on the two sides are physically equivalent, so we only need to work on one side.

\section{Some numerical results}

We have simulated the single-site model using the Metropolis algorithm and calculating the determinant (which is real and positive) exactly. CPU time scales as $N^{8}$ for a complete update of each link matrix, and we have been able to work only up to $N=15$ using desktop PCs. Nevertheless, this appears to be sufficient to map out the phase diagram, since the results do not change substantially between $N=8$ and $N=15$. For more details, see Ref. [6].

We have tested our conjecture for the phase diagram by doing horizontal hysteresis scans for couplings in the range $b=0.1-1$, vertical scans for a variety of values of $\kappa$, and a few high statistics runs at selected points in the putative funnel region. Very long correlation times limit our simulations to $b \leq 1$. We note, however, that $b=1$ is a very weak coupling, corresponding to $\beta=18$ in $S U(3)$.

We calculate the average plaquette as well as a number of order-parameter for $Z_{N}^{4}$ symmetrybreaking: Polyakov loops $P_{\mu}=\operatorname{Tr}\left(U_{\mu}\right) / N$ and "corner loops" $M_{\mu v}=\operatorname{Tr}\left(U_{\mu} U_{v}\right) / N$. In the momentumquenched EK model we found that one needed the $M_{\mu \nu}$ to uncover the symmetry-breaking [4], and we find the same here: there are regions of symmetry breaking in the $\kappa>\kappa_{c}$ part of the phaseplane for which the $P_{\mu}$ vanish but the $M_{\mu \nu}$ do not. To make sure that we do not miss any signals of symmetry breaking, we have, for a few points in the plane, also calculated the 14641 different traces

$$
K_{\vec{n}} \equiv \operatorname{Tr}\left(U_{1}^{n_{1}} U_{2}^{n_{2}} U_{3}^{n_{3}} U_{4}^{n_{4}}\right) / N, \quad \text { with } n_{\mu}=0, \pm 1, \pm 2, \ldots, \pm 5
$$



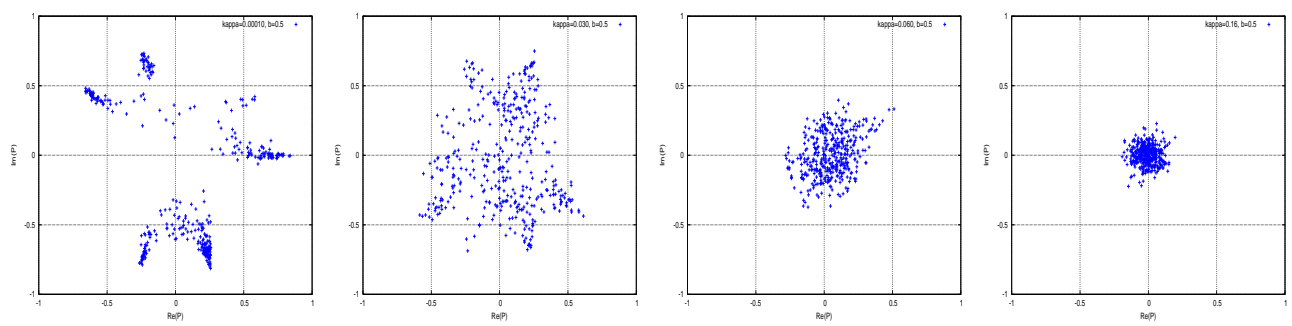

Figure 2: Scatter plots in the complex plane for all four Polyakov loops for $N=10, b=0.5$ and (moving from left to right) $\kappa=0.0001,0.03,0.06$ and 0.16 . Both horizontal and vertical scales run from -1 to 1 .

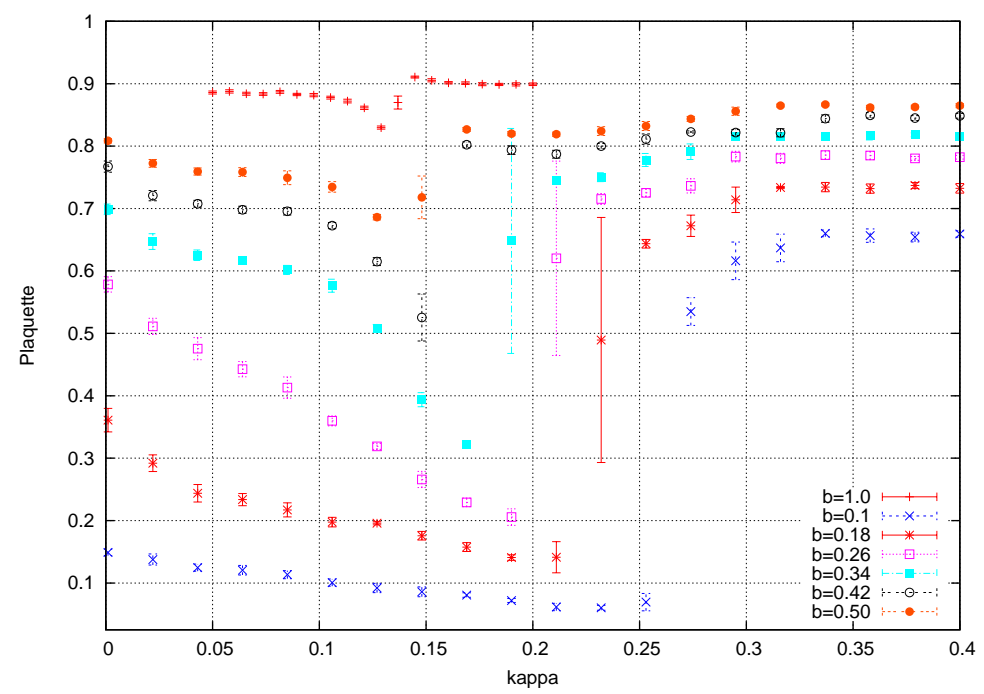

Figure 3: Scans of the plaquette as a function of $\kappa$ for $N=8$ at $b=0.1,0.18,0.26,0.34,0.42,0.50$ and 1 .

where $n_{\mu}<0$ implies hermitian conjugation.

Our results are consistent with the conjecture described above; see Ref. [6] for complete details. As an example, we show in Fig. 2 scatter plots of Polyakov loops for $N=10$ and $b=0.5$. For $\kappa=0$, symmetry breaking is clear: all four $P_{\mu}$ end up proportional to $e^{2 \pi i n_{\mu} / 10}$ (with, in this case, different $n_{\mu}$ ), although, for this small $N$, there is still some tunneling between different "vacua". Vestiges of symmetry breaking are still apparent at $\kappa=0.03$, but are absent at $\kappa=0.06$. For this value and for $\kappa=0.16$ (just below $\kappa_{c}$ ) the $P_{\mu}$ fluctuate around the origin and average to zero within errors. Results at $N=15$ are consistent, with the expected reduction in tunneling for small $\kappa$ (none is seen) and the expected reduction in the size of fluctuations.

Evidence for the presence of a first-order transition is shown in Fig. 3: there is a jump in the average plaquette for each value of $b$. We also observe hysteresis at this "transition", and a consistent behavior for $N=8-15$. The transition is not, however, associated with center symmetry breaking and we interpret it as an example of the first-order scenario predicted by chiral perturbation theory. This is supported by two other observations. First, its position is consistent with the theoretical expectations-moving from $1 / 4$ at strong coupling to $1 / 8$ at weak coupling. Second, the jump at the transition decreases as $b$ increases, consistent with the theoretical expectation that the transition is driven by lattice artefacts. 


\section{Conclusions and Outlook}

In summary, our evidence to date is consistent with the conjecture in the right panel of Fig. 1, including the presence of a window around $\kappa_{c}$ of finite width in which the $Z_{N}^{4}$ symmetry is unbroken. Accepting this result, the adjoint $N_{f}=1$ theory provides the first successful example of reduction to a single-site. Furthermore, it appears that the funnel region is broad enough to allow one to work with quarks with masses $m \gg \Lambda_{\mathrm{QCD}}$ (and possibly with $m \sim 1 / a$ ) so that the longdistance physics is that of the pure gauge theory. In this case we have, in effect, a version of the original EK model in which the effect of the adjoint quarks is to maintain the repulsion among eigenvalues of the Polyakov loop, presumably by inducing a tower of double-trace operators similar to that suggested in Ref. [14] as a cure for the EK model. If, on the other hand, we work near $\kappa_{c}$ we have a single site model that lies "within $1 / N$ " of physical two-flavor QCD.

Our analysis can be extended in several ways. Our interpretation of the critical line can be checked by calculating the pion mass. We can also calculate other meson masses and the string tension, and the distribution of the eigenvalues of Dirac operators for various valence fermions. Work in these directions is underway using the configurations in hand. We also plan on speeding up the algorithm so as to move to larger values of $N$.

Finally, we note that another recent lattice study (using overlap as opposed to our Wilson fermions) finds preliminary evidence for a failure of reduction [15]. This discrepancy with our findings will clearly need to be resolved.

\section{References}

[1] T. Eguchi and H. Kawai, Phys. Rev. Lett. 48, 1063 (1982).

[2] G. Bhanot, U. M. Heller and H. Neuberger, Phys. Lett. B 113, 47 (1982).

[3] V. A. Kazakov and A. A. Migdal, Phys. Lett. B 116, 423 (1982).

[4] B. Bringoltz and S. R. Sharpe, Phys. Rev. D 78, 034507 (2008) [arXiv:0805.2146 [hep-lat]].

[5] J. Kiskis, R. Narayanan and H. Neuberger, Phys. Rev. D 66, 025019 (2002) [arXiv:hep-lat/0203005].

[6] B. Bringoltz and S. R. Sharpe, arXiv:0906.3538 [hep-lat].

[7] E. Corrigan and P. Ramond, Phys. Lett. B 87, 73 (1979).

[8] A. Armoni, M. Shifman and G. Veneziano, Phys. Rev. Lett. 91, 191601 (2003) [arXiv:hep-th/0307097].

[9] P. Kovtun, M. Unsal and L. G. Yaffe, JHEP 0706, 019 (2007) [arXiv:hep-th/0702021].

[10] P. Kovtun, M. Unsal and L. G. Yaffe, JHEP 0507, 008 (2005) [arXiv:hep-th/0411177].

[11] T. J. Hollowood and J. C. Myers, arXiv:0907.3665 [hep-th].

[12] B. Bringoltz, JHEP 0906, 091 (2009) [arXiv:0905.2406 [hep-lat]].

[13] P. F. Bedaque, M. I. Buchoff, A. Cherman and R. P. Springer, arXiv:0904.0277 [hep-th].

[14] M. Unsal and L. G. Yaffe, Phys. Rev. D 78, 065035 (2008) [arXiv:0803.0344 [hep-th]].

[15] A. Hietanen and R. Narayanan, talk at this meeting. 